\title{
ON THE PERIODIC SOLUTIONS OF THE MILCHELSON CONTINUOUS AND DISCONTINUOUS PIECEWISE LINEAR DIFFERENTIAL SYSTEM
}

\author{
JAUME LLIBRE ${ }^{1}$, REGILENE OLIVEIRA² AND CAMILA A. B. \\ RODRIGUES $^{3}$
}

\begin{abstract}
Applying new results from the averaging theory for discontinuous and continuous differential systems, we study the periodic solutions of two distinct versions of the Michelson differential system: a Michelson continuous piecewise linear differential system, and a Michelson discontinuous piecewise linear differential system.
\end{abstract}

\section{IntRoduction AND STATEMENT OF THE MAIN RESUlt}

The Michelson differential system is given by

$$
\begin{aligned}
& \dot{x}=y, \\
& \dot{y}=z, \\
& \dot{z}=c^{2}-y-\frac{x^{2}}{2},
\end{aligned}
$$

with $(x, y, z) \in \mathbb{R}^{3}$ and the parameter $c \geq 0$. The dot denotes derivative with respect to an independent variable $t$, usually called the time. This system is due to Michelson [13] for studying the traveling solutions of the Kuramoto-Sivashinsky equation. It also arises in the analysis of the unfolding of the nilpotent singularity of codimension three $[4,6]$.

This system has been largely investigated from the dynamical point of view. In the first study of Michelson [13] he proved that if $c>0$ is sufficiently large, then system (1) has a unique bounded solution which is a transversal heteroclinic orbit connecting the two finite singularities $(-\sqrt{2} c, 0,0)$ and $(\sqrt{2} c, 0,0)$. When $c$ decreases there will appear a cocoon bifurcation (see $[7,8,13]$ ). A complete description of the phase portrait at infinity of system (1) via the Poincaré compactification was

2010 Mathematics Subject Classification. Primary: 34C29, 34C25, 37G15.

Key words and phrases. continuous piecewise linear differential systems, discontinuous piecewise linear differential systems, Michelson system, averaging theory, limit cycle, Hopf bifurcation. 
given in [14]. In [12] there is an analytical proof of the existence of a zero-Hopf bifurcation for system (1).

In $[3,2]$ the authors consider a continuous piecewise linear version of Michelson differential system changing the non linear function $x^{2}$ in (1) by the linear function $|x|$. For such system they proved that some dynamical aspects of the Michelson system remains as the existence of a reversible $\mathrm{T}$-point heteroclinic cycle.

Doing the change of variable $(x, y, z, c) \rightarrow(2 \varepsilon X, 2 \varepsilon Y, 2 \varepsilon Z, 2 \varepsilon d)$ with $d \geq 0$ and $\varepsilon>0$ sufficiently small to the differential Michelson system (1), followed by the change of the function $X^{2} \rightarrow|X|$, we obtain the system

$$
\begin{aligned}
& \dot{x}=y, \\
& \dot{y}=z, \\
& \dot{z}=-y+\varepsilon\left(2 d^{2}-|x|\right),
\end{aligned}
$$

that we call the Milchelson discontinuous piecewise linear differential system, where we still use $x, y, z$ instead of $X, Y, Z$.

In this paper first we study analytically the periodic solutions of the Michelson continuous piecewise linear differential system. Thus our first main result is the following.

Theorem 1. For all $d>0$ and $\varepsilon=\varepsilon(d)>0$ sufficiently small the Michelson continuous piecewise linear differential system (2) has a periodic solution of the form

$x(t)=-\pi d^{2}+\mathcal{O}(\varepsilon), \quad y(t)=\pi d^{2} \sin t+\mathcal{O}(\varepsilon), \quad z(t)=\pi d^{2} \cos t+\mathcal{O}(\varepsilon)$.

Moreover this periodic solution is linearly stable.

Theorem 1 is proved in section 3. Its proof uses an extension of the classical averaging theory for smooth differential systems to continuous differential systems given in [10], the first results in this continuous direction appeared in [1].

Many problems in physics, economics, biology and applied areas are modeled by discontinuous differential systems but there exist only few analytical techniques for studying their periodic solutions. In [11] the authors extended the averaging theory to discontinuous differential systems. An improvement of this result for a much bigger class of discontinuous differential systems is given in [9].

Applying these tools we investigate the periodic solutions of the Milchelson discontinuous piecewise linear differential system. In this version of the Michelson system we do the change of variable $(x, y, z, c) \rightarrow$ 
$(2 \varepsilon X, 2 \varepsilon Y, 2 \varepsilon Z, 2 \varepsilon d)$ with $d \geq 0$ and $\varepsilon>0$ sufficiently small to the differential Michelson system (1), and then the nonlinear function $x^{2}$ is changed by the discontinuous piecewise function

$$
f(x)=x+\operatorname{sign}(x),
$$

where here

$$
\operatorname{sign}(x)=\left\{\begin{aligned}
-1 & \text { if } x \leq 0 \\
1 & \text { if } x \geq 0
\end{aligned}\right.
$$

In short we consider the Milchelson discontinuous piecewise linear differential system given by

$$
\begin{aligned}
& \dot{x}=y, \\
& \dot{y}=z, \\
& \dot{z}=-y+\varepsilon\left(2 d^{2}-f(x)\right) .
\end{aligned}
$$

Again we will work with $x, y, z$ instead of $X, Y, Z$. We get the following result on the periodic solutions of system (3).

Theorem 2. For $\varepsilon>0$ sufficiently small the Milchelson discontinuous piecewise linear differential system (3) satisfies the following statements.

(a) If $d \in\left(0, \sqrt{\frac{3 \sqrt{3}-\pi}{6 \pi}}\right)$, then it has the two periodic solutions $(x(t, \varepsilon), r(t, \varepsilon), \theta(t, \varepsilon))$ given by

$$
\left(-r_{0}^{ \pm} \pm \frac{1}{4} \sqrt{16\left(r_{0}^{ \pm}\right)^{2}-\pi^{2}\left(r_{0}^{ \pm}\right)^{4}},\left(r_{0}^{ \pm}\right) \sin t,\left(r_{0}^{ \pm}\right) \cos t\right)+\mathcal{O}(\varepsilon),
$$

one with + and the other with - , where $r_{0}^{-}(d) \in(0,0.742953263 .$. and $r_{0}^{+}(d) \in(0.742953263 . ., 2 \sqrt{3} / \pi)$.

(b) If $d \in\left[\sqrt{\frac{3 \sqrt{3}-\pi}{6 \pi}}, \frac{1}{\sqrt{2}}\right)$, then it has only the above periodic solution with $r_{0}^{-}$.

These periodic solutions are linearly stable.

Theorem 2 is proved in section 4 .

\section{Preliminary}

For proving Theorems 1 and 2 we apply two recent results from the averaging theory, one for the continuous piecewise linear differential systems, and the other for the discontinuous piecewise linear differential systems. In this section we present these results and some remarks necessary for their applications. 
2.1. Continuous piecewise linear differential systems. From Theorem $\mathrm{B}$ of [10] we get the following result adapted to the next system (4).

Theorem 3. Consider the following system

$$
\dot{x}(t)=F_{0}(t, x)+\varepsilon F_{1}(t, x)+\varepsilon^{2} R(t, x, \varepsilon),
$$

where $F_{i}: \mathbb{R} \times D \rightarrow \mathbb{R}^{n}$ for $i=0,1$ and $R: \mathbb{R} \times D \times\left(-\varepsilon_{0}, \varepsilon_{0}\right) \rightarrow \mathbb{R}^{n}$ and for each $t \in \mathbb{R}$ the functions $F_{0}(t,.) \in C^{1}, F_{1}(t,.) \in C^{0}$ and $D_{x} F_{0}$ is locally Lipschitz in the second variable, and $R \in C^{0}$ and locally Lipschitz in the second variable. Moreover $D \subset \mathbb{R}^{n}$ is an open subset and $\varepsilon$ is a small parameter. Consider $\varphi(., z):\left[0, t_{z}\right] \rightarrow \mathbb{R}^{n}$ the solution of the unperturbed system such that $\varphi(0, z)=z$. Denote by $M(z)$ the fundamental solution matrix of the variational equation

$$
\dot{x}(t)=F_{0}(t, x),
$$

such that $M(0)$ is the identity. Assume that there exists an open and bounded subset of $V$ with its closure $\bar{V} \subset D$ such that for each $z \in \bar{V}$, the solution $x(t, z)$ is $T$ - periodic and satisfies $x(0, z)=z$. If $a \in V$ is a zero of the map $\mathcal{F}: \bar{V} \rightarrow \mathbb{R}^{n}$ defined by

$$
\mathcal{F}(z)=\int_{0}^{T} M^{-1}(t) F_{1}(t, x(t, z)) d t
$$

and $\operatorname{det}\left(D_{z} \mathcal{F}(a)\right) \neq 0$, then for $\varepsilon>0$ sufficiently small, system (4) has a T-periodic solution $x(t, \varepsilon)$ such that $x(0, \varepsilon) \rightarrow a$ as $\varepsilon \rightarrow 0$. Moreover the linear stability of the periodic solution $x(t, \varepsilon)$ is given by the eigenvalues of the matrix $D_{z} \mathcal{F}(a)$.

Note that the stability of the periodic solutions of system (4) when it is applied to the Michelson continuous piecewise linear differential system can be obtained from the stability of a differential system associated to it. In fact given the continuous system (1) consider a band of amplitude $\varepsilon>0$ around the plane $x=0$ and a differentiable extension of the continuous system (1) to this band. Studying the limit of this extended differentiable system when $\varepsilon \rightarrow 0$ we conclude that the linear stability of system (1) is given by the eigenvalues of $D_{z} \mathcal{F}(a)$.

2.2. Discontinuous piecewise linear differential systems. Let $D \subset$ $\mathbb{R}^{n}$ an open subset and $h: \mathbb{R} \times D \rightarrow \mathbb{R}$ a $C^{1}$ function having 0 as regular value. Consider $F^{1}, F^{2}: \mathbb{R} \times D \rightarrow \mathbb{R}^{n}$ continuous functions and $\Sigma=h^{-1}(0)$. We define the Filippov's system as

$$
\dot{x}(t)=F(t, x)= \begin{cases}F^{1}(t, x) & \text { if }(t, x) \in \Sigma^{+}, \\ F^{2}(t, x) & \text { if }(t, x) \in \Sigma^{-},\end{cases}
$$


where $\Sigma^{+}=\{(t, x) \in \mathbb{R} \times D: h(t, x)>0\}$ and $\Sigma^{-}=\{(t, x) \in \mathbb{R} \times D$ : $h(t, x)<0\}$.

The manifold $\Sigma$ is divided in the closure of two disjoint regions, namely Crossing region $\left(\Sigma^{c}\right)$ and Sliding region $\left(\Sigma^{s}\right)$,

$$
\begin{aligned}
& \Sigma^{c}=\left\{p \in \Sigma:\left\langle\nabla h(p),\left(1, F^{1}(p)\right)\right\rangle \cdot\left\langle\nabla h(p),\left(1, F^{2}(p)\right)\right\rangle>0\right\}, \\
& \Sigma^{s}=\left\{p \in \Sigma:\left\langle\nabla h(p),\left(1, F^{1}(p)\right)\right\rangle \cdot\left\langle\nabla h(p),\left(1, F^{2}(p)\right)\right\rangle<0\right\} .
\end{aligned}
$$

Consider the differential system associated to system (5)

$$
\dot{x}(t)=F(t, x)=\chi_{+}(t, x) F^{1}(t, x)+\chi_{-}(t, x) F^{2}(t, x),
$$

where $\chi_{+}, \chi_{-}$are the characteristic functions defined as

$$
\chi_{+}(t, x)=\left\{\begin{array}{l}
1 \text { if } h(t, x)>0 \\
0 \text { if } h(t, x)<0
\end{array}\right.
$$

and

$$
\chi_{-}(t, x)=\left\{\begin{array}{l}
0 \text { if } h(t, x)>0, \\
1 \text { if } h(t, x)<0 .
\end{array}\right.
$$

Systems (5) and (6) does not coincides in $h(t, x)=0$, but applying the Fillipov's convention for the solutions of systems (5) and (6) (see [5]) passing through a point $(t, x) \in \Sigma$ we have that these solutions do not depend on the value of $F(t, x)$, so the solutions are the same.

Let $\mathcal{P}$ be the space formed by the periodic solutions of (6). If $\operatorname{dim} \mathcal{P}=\operatorname{dim} D=d$ then the following result follows directly from Theorem B of [9].

Theorem 4. Consider the differential system

$$
\dot{x}(t)=F_{0}(t, x)+\varepsilon F_{1}(t, x)+\varepsilon^{2} R(t, x, \varepsilon) .
$$

where

$$
\begin{aligned}
F_{i}(t, x) & =\chi_{+} F_{i}^{1}(t, x)+\chi_{-} F_{i}^{2}(t, x), \text { for } i=0,1, \text { and } \\
R(t, x) & =\chi_{+} R^{1}(t, x)+\chi_{-} R^{2}(t, x),
\end{aligned}
$$

with $F_{i}^{1} \in C^{1}$, for $i=0,1$ and $R^{1}, R^{2}$ are continuous functions which are Lipschitz in the second variable, and all these functions are $T$ periodic functions in the variable $t \in \mathbb{R}$.

For $z \in D$ and $\varepsilon>0$ sufficiently small denote by $x(., z, \varepsilon):\left[0, t_{(z, \varepsilon)}\right] \rightarrow$ $\mathbb{R}^{d}$ the solution of system (7) such that $x(0, z, \varepsilon)=z$.

Define the averaged function

$$
\mathcal{F}(z)=\int_{0}^{T} M(s, z)^{-1} F_{1}(s, x(s, z, 0)) d s
$$


where $x(s, z, 0)$ is a periodic solution of (7) with $\varepsilon=0$ such that $x(0, z, 0)=z$, and $M(s, z)$ is the fundamental matrix of the variational system $\dot{y}=D_{x} F_{0}(t, x(t, z, 0)) y$ associated to the unperturbed system evaluated on the periodic solution $x(s, z, 0)$ such that $M(0, z)=I d$. Moreover we assume the following hypotheses.

$\left(H_{-}\right)$There exists an open bounded subset $C \subset D$ such that, for $\varepsilon$ sufficiently small, every orbit starting in $C$ reaches the set of discontinuity only at its crossing region.

$\left(H_{+}\right)$For $a \in C$ with $f(a)=0$ there exists a neighborhood $U \subset C$ of a such that $f(z) \neq 0$, for all $z \in \bar{U} \backslash\{a\}$ and $\operatorname{det}\left(D_{z} \mathcal{F}(a)\right) \neq 0$.

Then for $\varepsilon>0$ sufficiently small there exists a T-periodic solution $x(t, \varepsilon)$ of $(7)$ such that $x(0, \varepsilon) \rightarrow a$ as $\varepsilon \rightarrow 0$. Moreover the linear stability of the periodic solution $x(t, \varepsilon)$ is given by the eigenvalues of the matrix $D_{z} \mathcal{F}(a)$.

The same arguments for computing the kind of stability of the obtained periodic solution from the eigenvalues of the Jacobian matrix for the continuous piecewise differential systems, also work for the discontinuous piecewise differential systems.

\section{Proof of Theorem 1}

Doing to the Michelson continuous piecewise linear differential system (2) the change to cylindrical coordinates $x=x, y=r \sin \theta$ and $z=r \cos \theta$, then we have

$$
\begin{aligned}
\dot{x} & =r \sin \theta, \\
\dot{r} & =\varepsilon \cos \theta\left(2 d^{2}-|x|\right), \\
\dot{\theta} & =1-\frac{\varepsilon}{r} \sin \theta\left(2 d^{2}-|x|\right) .
\end{aligned}
$$

Taking $\theta$ as the new independent variable we obtain the system

$$
\begin{aligned}
& \frac{d x}{d \theta}=x^{\prime}=r \sin \theta+\varepsilon\left(2 d^{2}-|x|\right) \sin ^{2} \theta+\mathcal{O}\left(\varepsilon^{2}\right), \\
& \frac{d r}{d \theta}=r^{\prime}=\varepsilon\left(2 d^{2}-|x|\right) \cos \theta+\mathcal{O}\left(\varepsilon^{2}\right) .
\end{aligned}
$$

The unperturbed system is

$$
\begin{aligned}
x^{\prime} & =r \sin \theta, \\
r^{\prime} & =0 .
\end{aligned}
$$

For each $\left(x_{0}, r_{0}\right)$ the solution $\varphi\left(\theta,\left(x_{0}, r_{0}\right)\right)$ such that $\varphi\left(0,\left(x_{0}, r_{0}\right)\right)=$ $\left(x_{0}, r_{0}\right)$ is $\varphi\left(\theta,\left(x_{0}, r_{0}\right)\right)=\left(x_{0}+r_{0}(1-\cos \theta), r_{0}\right)$, which is $2 \pi-$ periodic 
for all $\left(x_{0}, r_{0}\right) \neq(0,0)$. When $\left(x_{0}, r_{0}\right)=(0,0)$ we have an equilibrium point.

Now note that the function $F_{0}(\theta,(x, r))=(r \sin \theta, 0)$ is $C^{\infty}$ and in particular $C^{1}$, and that the function

$$
F_{1}(\theta,(x, r))=\left(\left(2 d^{2}-|x|\right) \sin ^{2} \theta,\left(2 d^{2}-|x|\right) \cos \theta\right)
$$

is $C^{0}$, and both are Lipschitz. So the differential system (9) satisfies the assumptions of Theorem 3. Then, by Theorem 3, we need to calculate the averaged function

$$
\mathcal{F}\left(x_{0}, r_{0}\right)=\int_{0}^{2 \pi} M(\theta)^{-1} F_{1}\left(\theta, \varphi\left(\theta,\left(x_{0}, r_{0}\right)\right)\right) d \theta
$$

where

$$
M(\theta)=\left(\begin{array}{cc}
1 & 1-\cos \theta \\
0 & 1
\end{array}\right)
$$

is the fundamental matrix of the variational differential system associated to system (11) evaluated on the periodic solution $\left(x_{0}+r_{0}(1-\right.$ $\left.\cos \theta), r_{0}\right)$ such that $M(0)$ is the identity matrix. Therefore we have

$$
\begin{aligned}
\mathcal{F}\left(x_{0}, r_{0}\right) & =\int_{0}^{2 \pi}\left(\begin{array}{cc}
1 & \cos \theta-1 \\
0 & 1
\end{array}\right)\left(\begin{array}{c}
\left(2 d^{2}-\left|x_{0}+r_{0}(1-\cos \theta)\right|\right) \sin ^{2} \theta \\
\left(2 d^{2}-\left|x_{0}+r_{0}(1-\cos \theta)\right|\right) \cos \theta
\end{array}\right) d \theta \\
& =\int_{0}^{2 \pi}\left(\begin{array}{c}
\left(2 d^{2}-\left|x_{0}+r_{0}(1-\cos \theta)\right|\right)(1-\cos \theta) \\
\left(2 d^{2}-\left|x_{0}+r_{0}(1-\cos \theta)\right|\right) \cos \theta
\end{array}\right) d \theta \\
& =\int_{0}^{2 \pi} g(\theta) d \theta .
\end{aligned}
$$

Note that $g(\theta)=g(-\theta)$ and $g(\theta)$ is $2 \pi$-periodic. So

$$
\int_{0}^{2 \pi} g(\theta) d \theta=\int_{-\pi}^{\pi} g(\theta) d \theta=2 \int_{0}^{\pi} g(\theta) d \theta
$$

To calculate this integral we need to study the zeros of the function $G(\theta) \doteq x_{0}+r_{0}(1-\cos \theta)$.

As $G(\theta)=0$ if and only if $\theta= \pm \arccos \left(\frac{x_{0}+r_{0}}{r_{0}}\right)$ and the function $\arccos (x)$ takes real values when $x \in[-1,1]$ we have to consider the following cases.

Case 1: $\left|\frac{x_{0}+r_{0}}{r_{0}}\right|>1$. So $r_{0}+x_{0}-r_{0} \cos \theta \neq 0$ and we consider two subcases.

Subcase 1.1: $\frac{x_{0}+r_{0}}{r_{0}}<-1$. Then $r_{0}+x_{0}-r_{0} \cos \theta<0$ in $[0, \pi]$.

Subcase 1.2: $\frac{x_{0}+r_{0}}{r_{0}}>1$. Then $r_{0}+x_{0}-r_{0} \cos \theta>0$ in $[0, \pi]$. 
Case 2: $\left|\frac{x_{0}+r_{0}}{r_{0}}\right| \leq 1$. In this case $\theta=\arccos \left(\frac{r_{0}+x_{0}}{r_{0}}\right) \in[0, \pi]$ is the unique zero of $G(\theta)$. So we need to divide this analysis in two subcases: Subcase 2.1: $r_{0}+x_{0} \leq 0$. Then

(i) $r_{0}+x_{0}-r_{0} \cos \theta<0$, if $\theta \in\left(0, \arccos \left(\frac{r_{0}+x_{0}}{r_{0}}\right)\right)$,

(ii) $r_{0}+x_{0}-r_{0} \cos \theta>0$, if $\theta \in\left(\arccos \left(\frac{r_{0}+x_{0}}{r_{0}}\right), \pi\right)$.

Subcase 2.2: $r_{0}+x_{0}>0$. Then

(i) $r_{0}+x_{0}-r_{0} \cos \theta>0$, if $\theta \in\left(0, \arccos \left(\frac{r_{0}+x_{0}}{r_{0}}\right)\right)$,

(ii) $r_{0}+x_{0}-r_{0} \cos \theta<0$ if $\theta \in\left(\arccos \left(\frac{r_{0}+x_{0}}{r_{0}}\right), \pi\right)$.

In the computation of the integral $\int_{0}^{\pi} g(\theta) d \theta$ we need to distinguish the previous four subcases.

Subcase 1.1. In this subcase the averaged function is

$$
\begin{aligned}
\mathcal{F}\left(x_{0}, r_{0}\right) & =2 \int_{0}^{\pi}\left(\begin{array}{c}
\left(2 d^{2}+\left(x_{0}+r_{0}(1-\cos \theta)\right)(1-\cos \theta)\right. \\
\left(2 d^{2}+\left(x_{0}+r_{0}(1-\cos \theta)\right) \cos \theta\right.
\end{array}\right) d \theta \\
& \left.=\pi\left(4 d^{2}+3 r_{0}+2 x_{0}\right),-\pi r_{0}\right),
\end{aligned}
$$

whose unique zero is $\left(x_{0}, r_{0}\right)=\left(-2 d^{2}, 0\right)$. Since this initial condition corresponds to an equilibrium point of the unperturbed system (11), the averaging theory in this case does not provide periodic solutions.

Subcase 1.2. Analogously to Subcase 1.1 we have

$$
\begin{aligned}
\mathcal{F}\left(x_{0}, r_{0}\right) & =2 \int_{0}^{\pi}\left(\begin{array}{c}
\left(2 d^{2}+\left(x_{0}+r_{0}(1-\cos \theta)\right)(1-\cos \theta)\right. \\
\left(2 d^{2}+\left(x_{0}+r_{0}(1-\cos \theta)\right) \cos \theta\right.
\end{array}\right) d \theta \\
& \left.=\pi\left(4 d^{2}-3 r_{0}-2 x_{0}\right), \pi r_{0}\right)
\end{aligned}
$$

whose unique zero is $\left(x_{0}, r_{0}\right)=\left(2 d^{2}, 0\right)$. The conclusion follows as in Subcase 1.1.

Subcase 2.1. Here

$$
\begin{aligned}
\mathcal{F}\left(x_{0}, r_{0}\right)= & 2 \int_{0}^{\arccos \left(\frac{r_{0}+x_{0}}{r_{0}}\right)}\left(\begin{array}{c}
\left(2 d^{2}+\left(x_{0}+r_{0}(1-\cos \theta)\right)(1-\cos \theta)\right. \\
\left(2 d^{2}+\left(x_{0}+r_{0}(1-\cos \theta)\right) \cos \theta\right.
\end{array}\right) d \theta \\
& +2 \int_{\arccos \left(\frac{r_{0}+x_{0}}{r_{0}}\right)}^{\pi}\left(\begin{array}{c}
\left(2 d^{2}-\left(x_{0}+r_{0}(1-\cos \theta)\right)\right)(1-\cos \theta) \\
\left(2 d^{2}-\left(x_{0}+r_{0}(1-\cos \theta)\right)\right) \cos \theta
\end{array}\right) d \theta \\
= & \left(\begin{array}{c}
f_{1}\left(x_{0}, r_{0}\right) \\
f_{2}\left(x_{0}, r_{0}\right)
\end{array}\right),
\end{aligned}
$$


where

$$
\begin{gathered}
f_{1}\left(x_{0}, r_{0}\right)=4 d^{2} \pi-\frac{\sqrt{-x_{0}\left(2 r_{0}+x_{0}\right)}}{r_{0}}\left(6 r_{0}+2 x_{0}\right) \\
-\pi\left(3 r_{0}+2 x_{0}\right)+\left(6 r_{0}+4 x_{0}\right) \arccos \left(\frac{r_{0}+x_{0}}{r_{0}}\right), \\
f_{2}\left(x_{0}, r_{0}\right)=\pi r_{0}+2\left(r_{0}+x_{0}\right) \frac{\sqrt{-x_{0}\left(2 r_{0}+x_{0}\right)}}{r_{0}} \\
-2 r_{0} \arccos \left(\frac{r_{0}+x_{0}}{r_{0}}\right) .
\end{gathered}
$$

Claim: The unique real root of this function is $\left(x_{0}, r_{0}\right)=\left(-d^{2} \pi, d^{2} \pi\right)$.

Proof. In fact, calling $A=\arccos \left(\frac{x_{0}+r_{0}}{r_{0}}\right)$ then $f_{2}\left(x_{0}, r_{0}\right)=0$ if and only if $A=\frac{1}{2 r_{0}}\left(\pi r_{0}+\frac{2\left(r_{0}+x_{0}\right)}{r_{0}} \sqrt{-x_{0}\left(2 r_{0}+x_{0}\right)}\right)$.

Replacing the expression of $A$ in (12) we obtain

$$
\left.f_{1}\left(x_{0}, y_{0}\right)\right|_{A}=4 d^{2} \pi-4 r_{0}\left(-\frac{x_{0}\left(2 r_{0}+x_{0}\right)}{r_{0}^{2}}\right)^{\frac{3}{2}} \text {. }
$$

The function $\left.f_{1}\left(x_{0}, y_{0}\right)\right|_{A}$ has two real zeros if $-d^{4 / 3} \pi^{2 / 3} r_{0}^{4 / 3}+r_{0}^{2} \geq 0$, namely,

$$
x_{0}=-r_{0} \pm \sqrt{-d^{4 / 3} \pi^{2 / 3} r_{0}^{4 / 3}+r_{0}^{2}} .
$$

Now if we replace $x_{0}=-r_{0}-\sqrt{-d^{4 / 3} \pi^{2 / 3} r_{0}^{4 / 3}+r_{0}^{2}}$ in (13) we obtain

$$
\begin{aligned}
f_{2}\left(r_{0}\right)= & -\frac{2 \pi^{1 / 3} d^{2 / 3} \sqrt{-d^{4 / 3} \pi^{2 / 3} r_{0}^{4 / 3}+r_{0}^{2}}}{r_{0}^{1 / 3}} \\
& -2 r_{0} \operatorname{arccsc}\left(\frac{r_{0}}{\sqrt{-d^{4 / 3} \pi^{2 / 3} r_{0}^{4 / 3}+r_{0}^{2}}}\right) .
\end{aligned}
$$

Since the function $\operatorname{arccsc}(z)>0$ for all $z>0$ and goes to 0 when $z \rightarrow \infty$ we have that

$$
-2 r_{0} \operatorname{arccsc}\left(\frac{r_{0}}{\sqrt{-d^{4 / 3} \pi^{2 / 3} r_{0}^{4 / 3}+r_{0}^{2}}}\right)<0,
$$


and taking into account that

$$
-\frac{2 \pi^{1 / 3} d^{2 / 3} \sqrt{-d^{4 / 3} \pi^{2 / 3} r_{0}^{4 / 3}+r_{0}^{2}}}{r_{0}^{1 / 3}} \leq 0
$$

for all $r_{0}>0$, then the unique possibility in order that $f_{2}\left(r_{0}\right)$ to be zero, is when $-d^{4 / 3} \pi^{2 / 3} r_{0}^{4 / 3}+r_{0}^{2} \rightarrow 0$, i. e. $r_{0} \rightarrow d^{2} \pi$.

Moreover if $r_{0} \rightarrow d^{2} \pi$ then $x_{0} \rightarrow-d^{2} \pi$, and we have a candidate to zero of the averaged function, which is a zero because $\mathcal{F}\left(-d \pi^{2}, d \pi^{2}\right)=0$ by direct computations. This completes the proof of the claim.

The Jacobian of the map $\mathcal{F}\left(x_{0}, r_{0}\right)$ evaluated at $\left(x_{0}, r_{0}\right)=\left(-d^{2} \pi, d^{2} \pi\right)$ is

$$
\left|\begin{array}{cc}
8 & -4 \\
4 & 4
\end{array}\right|=16 \neq 0
$$

It follows from Theorem 3 and for any $d>0$ and $\varepsilon=\varepsilon(d)>0$ sufficiently small that system (10) has a periodic solution $\varphi(\theta, \varepsilon)=$ $(x(\theta, \varepsilon), r(\theta, \varepsilon))=\left(-d^{2} \pi+\mathcal{O}(\varepsilon), d^{2} \pi+\mathcal{O}(\varepsilon)\right)$. Moreover the eigenvalues of the Jacobian matrix of $\mathcal{F}\left(x_{0}, r_{0}\right)$ at the solution $\left(-d^{2} \pi, d^{2} \pi\right)$ are $\pm 4 i$, so the periodic solution is linearly stable.

Subcase 2.2. In this subcase

$$
\begin{aligned}
\mathcal{F}\left(x_{0}, r_{0}\right)= & 2 \int_{0}^{\arccos \left(\frac{r_{0}+x_{0}}{r_{0}}\right)}\left(\begin{array}{c}
\left(2 d^{2}-\left(x_{0}+r_{0}(1-\cos \theta)\right)(1-\cos \theta)\right. \\
\left(2 d^{2}-\left(x_{0}+r_{0}(1-\cos \theta)\right) \cos \theta\right.
\end{array}\right) d \theta \\
& +2 \int_{\arccos \left(\frac{r_{0}+x_{0}}{r_{0}}\right)}^{\pi}\left(\begin{array}{c}
\left(2 d^{2}+\left(x_{0}+r_{0}(1-\cos \theta)\right)\right)(1-\cos \theta) \\
\left(2 d^{2}+\left(x_{0}+r_{0}(1-\cos \theta)\right)\right) \cos \theta
\end{array}\right) d \theta \\
= & \left(\begin{array}{c}
g_{1}\left(x_{0}, r_{0}\right) \\
g_{2}\left(x_{0}, r_{0}\right)
\end{array}\right),
\end{aligned}
$$

where

$$
\begin{aligned}
g_{1}\left(x_{0}, r_{0}\right)= & 4 d^{2} \pi+\frac{\sqrt{-x_{0}\left(2 r_{0}+x_{0}\right)}}{r_{0}}\left(6 r_{0}+2 x_{0}\right) \\
& +\left(6 r_{0}+4 x_{0}\right) \arccos \left(\frac{r_{0}+x_{0}}{r_{0}}\right) \\
g_{2}\left(x_{0}, r_{0}\right)= & -\pi r_{0}-2\left(r_{0}+x_{0}\right) \frac{\sqrt{-x_{0}\left(2 r_{0}+x_{0}\right)}}{r_{0}} \\
& +2 r_{0} \arccos \left(\frac{r_{0}+x_{0}}{r_{0}}\right) .
\end{aligned}
$$

If we work as in Subcase 2.1 we will find that the only possibility for the vanishing of this function is when $x_{0}=-r_{0}$ with $r_{0}<0$. Hence $\mathcal{F}\left(x_{0}, r_{0}\right)$ has no solutions with $r_{0}>0$. 
Now we must identify the periodic solution of system (2) which corresponds to the periodic solution found in Subcase 2.1. Going back to system (9) with the independent variable $t$ we obtain the periodic solution

$$
(x(t, \varepsilon), r(t, \varepsilon), \theta(t, \varepsilon))=\left(-d^{2} \pi, d^{2} \pi, t(\bmod 2 \pi)\right)+\mathcal{O}(\varepsilon) .
$$

Finally coming back to system (2) we find the periodic solution

$$
(x(t, \varepsilon), y(t, \varepsilon), z(t, \varepsilon))=\left(\left(-d^{2} \pi, d^{2} \pi \sin t, d^{2} \pi \cos t\right)+\mathcal{O}(\varepsilon) .\right.
$$

This concludes the proof of Theorem 1.

\section{Proof of Theorem 2}

Doing the change to cylindrical coordinates $x=x, y=r \sin \theta$ and $z=r \cos \theta$ the Michelson discontinuous piecewise linear differential system becomes

$$
\begin{aligned}
& \dot{x}=r \sin \theta, \\
& \dot{r}=\varepsilon \cos \theta\left(2 d^{2}-f(x)\right), \\
& \dot{\theta}=1-\frac{\varepsilon}{r} \sin \theta\left(2 d^{2}-f(x)\right) .
\end{aligned}
$$

Now taking as new independent variable the angle $\theta$ we get the system

$$
\begin{aligned}
& x^{\prime}=r \sin \theta+\varepsilon\left(2 d^{2}-(x+\operatorname{sign}(x))\right)(1-\cos \theta)+\mathcal{O}\left(\varepsilon^{2}\right), \\
& r^{\prime}=\varepsilon\left(2 d^{2}-(x+\operatorname{sign}(x))\right) \cos \theta+\mathcal{O}\left(\varepsilon^{2}\right),
\end{aligned}
$$

where the prime denotes the derivative with respect to $\theta$.

The unperturbed system is given by (11) and the fundamental matrix is $M(\theta)=\left(\begin{array}{cc}1 & 1-\cos \theta \\ 0 & 1\end{array}\right)$. Then by Theorem 4 we need to calculate

$$
\mathcal{F}\left(x_{0}, r_{0}\right)=\int_{0}^{2 \pi} g(\theta) d \theta .
$$

where

$g(\theta)=\left(\begin{array}{c}\left(2 d^{2}-\left(x_{0}+r_{0}(1-\cos \theta)+\operatorname{sign}\left(x_{0}+r_{0}(1-\cos \theta)\right)\right)(1-\cos \theta)\right. \\ \left(2 d^{2}-\left(x_{0}+r_{0}(1-\cos \theta)+\operatorname{sign}\left(x_{0}+r_{0}(1-\cos \theta)\right)\right) \cos \theta\right.\end{array}\right)$.

Since $g(\theta)$ is $2 \pi$-periodic and $g(\theta)=g(-\theta)$ then

$$
\int_{0}^{2 \pi} g(\theta) d \theta=2 \int_{0}^{\pi} g(\theta) d \theta
$$

Analogously to the study of the continuous system, we separate the calculation of the averaged function in four subcases, the same ones as in Theorem 3. 
Subcase 1.1. In this subcase $r_{0}+x_{0}-r_{0} \cos \theta<0$ in $[0, \pi]$. Then the averaged function, computed using formula (8), is

$$
\mathcal{F}\left(x_{0}, r_{0}\right)=\left(\pi\left(2+4 d^{2}-3 r_{0}-2 x_{0}\right), \pi r_{0}\right),
$$

whose unique zero is $\left(x_{0}, r_{0}\right)=\left(1+2 d^{2}, 0\right)$. Since this initial condition correspond to an equilibrium point of the unperturbed system (11), the averaging theory in this case does not produce any periodic solution.

Subcase 1.2. Now analogously to Subcase 1.1 we have

$$
f\left(x_{0}, r_{0}\right)=\left(\pi\left(-2+4 d^{2}-3 r_{0}-2 x_{0}\right), \pi r_{0}\right),
$$

whose only zero is $\left(x_{0}, r_{0}\right)=\left(-1+2 d^{2}, 0\right)$. The conclusion follows as in Subcase 1.1.

Subcase 2.1. Here $r_{0}+x_{0}-r_{0} \cos \theta<0$ when $\theta \in\left(0, \arccos \left(\frac{r_{0}+x_{0}}{r_{0}}\right)\right)$, and $r_{0}+x_{0}-r_{0} \cos \theta>0$, when $\theta \in\left(\arccos \left(\frac{r_{0}+x_{0}}{r_{0}}\right), \pi\right)$, so

$$
\mathcal{F}\left(x_{0}, r_{0}\right)=\left(\begin{array}{c}
f_{1}\left(x_{0}, r_{0}\right) \\
f_{2}\left(x_{0}, r_{0}\right)
\end{array}\right)
$$

with

$$
\begin{aligned}
f_{1}\left(x_{0}, r_{0}\right)= & -4 \frac{\sqrt{-\left(x_{0}\left(2 r_{0}+x_{0}\right)\right)}}{r_{0}}-4 \arccos \left(\frac{x_{0}+r_{0}}{r_{0}}\right)+ \\
& +4 d^{2} \pi-3 \pi r_{0}-2 \pi x_{0}, \\
f_{2}\left(x_{0}, r_{0}\right)= & \pi r_{0}+4 \frac{\sqrt{-x_{0}\left(2 r_{0}+x_{0}\right)}}{r_{0}} .
\end{aligned}
$$

Note $f_{2}\left(x_{0}, r_{0}\right)=0$ if and only if $r_{0}^{2}=-\frac{4}{\pi} \sqrt{-x_{0}\left(2 r_{0}+x_{0}\right)}<0$, which has no real solutions.

Subcase 2.2. In this case

$$
\mathcal{F}\left(x_{0}, r_{0}\right)=\left(\begin{array}{l}
f_{1}\left(x_{0}, r_{0}\right) \\
f_{2}\left(x_{0}, r_{0}\right)
\end{array}\right),
$$

where

$$
\begin{gathered}
f_{1}\left(x_{0}, r_{0}\right)=4 \frac{\sqrt{-x_{0}\left(2 r_{0}+x_{0}\right)}}{r_{0}}-4 \arccos \left(\frac{x_{0}+r_{0}}{r_{0}}\right)+ \\
+\pi\left(2+4 d^{2}-3 r_{0}-2 x_{0}\right) \\
f_{2}\left(x_{0}, r_{0}\right)=\pi r_{0}-4 \frac{\sqrt{-x_{0}\left(2 r_{0}+x_{0}\right)}}{r_{0}} .
\end{gathered}
$$


Note that $f_{2}\left(x_{0}, r_{0}\right)=0$ if and only if $x_{0}=-r_{0} \pm \frac{1}{4} \sqrt{16 r_{0}^{2}-\pi^{2} r_{0}^{4}}$. So these zeros exist if and only if $r_{0} \in\left(0, \frac{4}{\pi}\right)$.

Doing $x_{0}=-r_{0}-\frac{1}{4} \sqrt{16 r_{0}^{2}-\pi^{2} r_{0}^{4}}$ in (16) we get

$$
\begin{aligned}
\overline{f_{1}}\left(r_{0}\right) & =\left.f_{1}\left(x_{0}, r_{0}\right)\right|_{x_{0}=-r_{0}-\frac{1}{4} \sqrt{16 r_{0}^{2}-\pi^{2} r_{0}^{4}}} \\
& =4 d^{2} \pi+\frac{1}{2} \pi r_{0} \sqrt{16-\pi^{2} r_{0}^{2}}-4 \operatorname{arccsc}\left(\frac{4}{\sqrt{16-\pi^{2} r_{0}^{2}}}\right) .
\end{aligned}
$$

Define $H_{-}\left(r_{0}\right)=\overline{f_{1}}\left(r_{0}\right)-4 d^{2} \pi$. Figure 1 is the graphic of the function $H_{-}\left(r_{0}\right)$ when $r_{0} \in\left(0, \frac{4}{\pi}\right)$. From this graphic we conclude that the existence of zeros of $\overline{f_{1}}\left(r_{0}\right)$ depends on $d$. If such a zero $r_{0}^{-}$exists then it is unique. So it follows easily that $r_{0}^{-} \in(0,0.742953263 .$.$) exists if$ and only if $d \in\left(0, \frac{1}{\sqrt{2}}\right)$.

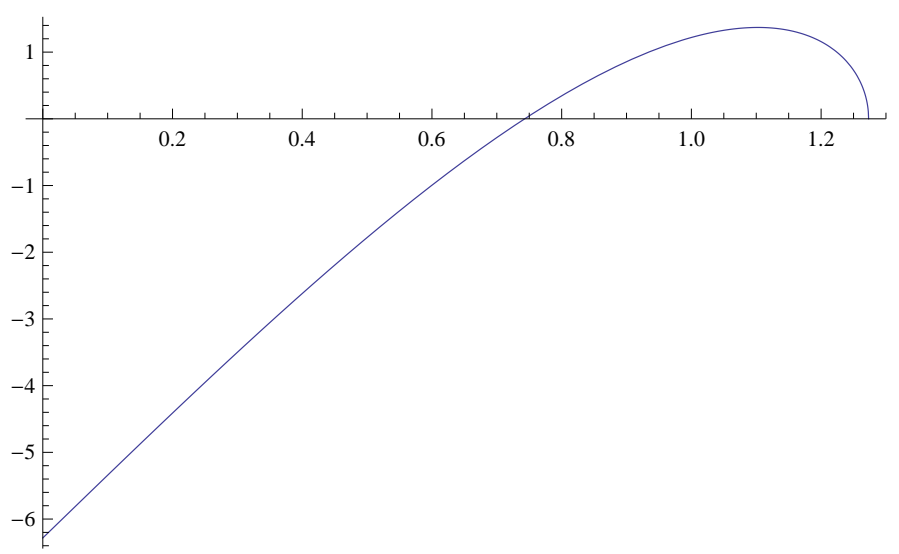

Figure 1. Graphic of $H_{-}\left(r_{0}\right)$

Now taking $x_{0}=-r_{0}+\frac{1}{4} \sqrt{16 r_{0}^{2}-\pi^{2} r_{0}^{4}}$ in (16) we get

$$
\begin{aligned}
& \overline{f_{1}}\left(r_{0}\right)=f_{1}\left(x_{0}, r_{0}\right) \mid x_{0}=-r_{0}+\frac{1}{4} \sqrt{16 r_{0}^{2}-\pi^{2} r_{0}^{4}} \\
& =4 d^{2} \pi-\frac{1}{2} \pi r_{0} \sqrt{16-\pi^{2} r_{0}^{2}}+4 \operatorname{arccsc}\left(\frac{4}{\sqrt{16-\pi^{2} r_{0}^{2}}}\right) .
\end{aligned}
$$


Again defining $H_{+}\left(r_{0}\right)=\overline{f_{1}}\left(r_{0}\right)-4 d^{2} \pi$ and plotting its graphic (see Figure 2) we can see that if we find the minimum of $H_{+}\left(r_{0}\right)$ we will be able to provide the condition in $d$ for the existence of zeros of $\overline{f_{1}}\left(r_{0}\right)$.

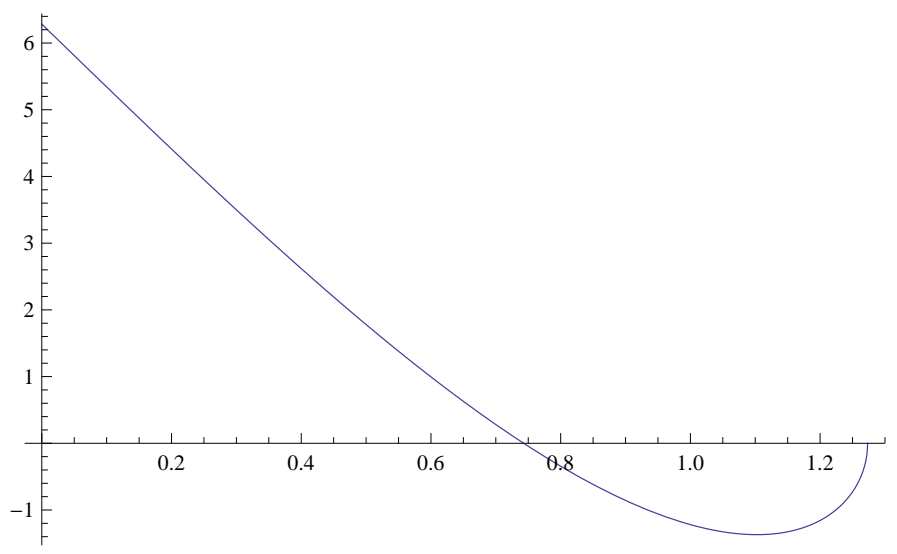

Figure 2. Graphic of $H_{+}\left(r_{0}\right)$

As $H_{+}^{\prime}\left(r_{0}\right)=\frac{\pi\left(\pi^{2} r_{0}^{2}-12\right)}{\sqrt{16-\pi^{2} r_{0}^{2}}}$, it follows that the minimum of $H_{+}\left(r_{0}\right)$ takes place at $r_{0}=2 \sqrt{3} / \pi$. Hence $\overline{f_{1}}\left(r_{0}\right)$ has a simple zero $r_{0}^{+} \in$ $(0.742953263 . ., 2 \sqrt{3} / \pi)$ if and only if $d \in\left(0, \sqrt{\frac{3 \sqrt{3}-\pi}{6 \pi}}\right)$. Moreover this zero is unique. So from Theorem 4 it follows

(i) if $d \in\left(0, \sqrt{\frac{3 \sqrt{3}-\pi}{6 \pi}}\right)$, then system (15) has at least two periodic solutions;

(ii) if $d \in\left(\sqrt{\frac{3 \sqrt{3}-\pi}{6 \pi}}, \frac{1}{\sqrt{2}}\right)$ then system (15) has at least one periodic solution; and

(iii) if $d \in\left(\frac{1}{\sqrt{2}}, \infty\right)$, we cannot apply the averaging theory for finding periodic solutions of system (15).

Going back to the independent variable $t$ for system (14) we conclude that there exist at least two periodic solutions $(x(t, \varepsilon), r(t, \varepsilon), \theta(t, \varepsilon))$ of system (14), namely

$$
\left(-r_{0}^{ \pm} \pm \frac{1}{4} \sqrt{16\left(r_{0}^{ \pm}\right)^{2}-\pi^{2}\left(r_{0}^{ \pm}\right)^{4}}, r_{0}^{ \pm}, t(\bmod 2 \pi)\right)+\mathcal{O}(\varepsilon),
$$


one with + and the other with - . Finally coming back to the Michelson discontinuous piecewise linear differential system (3) we get the two periodic solutions $(x(t, \varepsilon), r(t, \varepsilon), \theta(t, \varepsilon))$ given by

$$
\left(-r_{0}^{ \pm} \pm \frac{1}{4} \sqrt{16\left(r_{0}^{ \pm}\right)^{2}-\pi^{2}\left(r_{0}^{ \pm}\right)^{4}}, r_{0}^{ \pm} \sin t, r_{0}^{ \pm} \cos t\right)+\mathcal{O}(\varepsilon)
$$

Since $\pm 2 \sqrt{r_{0}\left(-12+\pi^{2} r_{0}^{2}\right)} / r_{0}^{3 / 2}$ are the eigenvalues of the Jacobian matrix of $\mathcal{F}\left(x_{0}, r_{0}\right)$ at the solutions $\left(x_{0}, r_{0}\right)=\left(-r_{0} \pm \frac{1}{4} \sqrt{16 r_{0}^{2}-\pi^{2} r_{0}^{4}}, r_{0}\right)$, and $0<r_{0}^{ \pm}<2 \sqrt{3} / \pi$, both periodic solutions of system (3) are linearly stable. This completes the proof of Theorem 2 .

\section{ACKNOWLEDGEMENTS}

The first author is partially supported by a MINECO/FEDER grant MTM2008-03437 and MTM2013-40998-P, an AGAUR grant number 2014SGR568, an ICREA Academia, the grants FP7-PEOPLE-2012IRSES 318999 and 316338, FEDER-UNAB-10-4E-378, and a CAPES grant number 88881.030454/2013-01 from the program CSF-PVE. The second authors is partially supported by the joint projects FP7-PEOPLE2012-IRSES numbers 316338, CAPES grant number 88881.030454/201301 from the program CSF-PVE and CNPq grant "Projeto Universal 472796/2013-5". The third author is supported by a FAPESP grant number 2012/22000-0.

\section{REFERENCES}

[1] A. Buica And J. LliBRe, Averaging methods for finding periodic orbits via Brouwer degree, Bull. Sci. Math. 128 (2004), 7-22.

[2] V. Carmona, F.Fernandez-Sánchez, E. García-Medina And A. TERUEL, Existence of homoclinic connections in continuous piecewise linear systems, Chaos 20 (2010), 013124, 8 pp.

[3] V. Carmona, F.Fernandez-Sánchez And A. Teruel, Existence of a Reversible T-Point Heteroclinic Cycle in a Piecewise Linear Version of the Michelson System, SIAM J. Applied Dynamical Systems 7 (2008), 1032-1048.

[4] F. Dumortier, S. Ibañez And H. Kokubu, New aspects in the unfolding of the nilpotent singularity of codimension three, Dyn. Syst. 16 (2001), 63-95.

[5] A. F. Filippov, Differential equations with discontinuous righthand side. Mathematics and Its Applications, Kluwer Academic Publishers, Dordrecht, 1988.

[6] E. Freire, E. Gamero, A. J. Rodriguez-Luis and A. Algaba, A note on the triple-zero linear degeneracy: Normal forms, dynamical and bifurcation behaviors of an unfolding, Internat. J. Bifur. Chaos 12 (2002), 2799-2820.

[7] H. Kokubu, D. Wilczak and P. ZGLiczyŃski, Rigorous verification of cocoon bifurcations in the Michelson system, Nonlinearity 20 (2007) 21472174 . 
[8] Y.-T LAU, The 'cocoon' bifurcation in three-dimensional systems with two fixed points, Internat. J. Bif. Chaos 2 (1992), 543-558.

[9] J. Llibre AND D. Novaes, On the periodic solutions of discontinous piecewise differential systems, Preprint 2014.

[10] J. Llibre, D. Novaes And M. A. Teixeira, Higher order averaging theory for finding periodic solutions via Brouwer degree, Nonlinearity 27 (2014), 563583.

[11] J. Llibre, D. Novaes And M.A.Teixeira, On the birth of limit cycles for non-smooth dynamical systems, to appear in Bull. Sci. Math.

[12] J. Llibre AND X. Zhang, On the Hopf-zero bifurcation of the Michelson system, Nonlinear Analysis: Real and World Appl. 12 (2011), 1650-1653.

[13] D. Michelson, Steady solutions for the Kuramoto-Sivashinsky equation, Physica D 19 (1986), 89-111.

[14] R. Oliveira And C. VAlls, Dynamics at infinity of Michelson, Lü and Tsystems, Preprint 2014.

${ }^{1}$ Departament de Matemàtiques, Universitat Autònoma de Barcelona, 08193 Bellaterra, Barcelona, Catalonia, Spain

E-mail address: jllibre@mat.uab.cat

$2,{ }^{3}$ Departamento de Matemática, ICMC-Universidade de São Paulo, C.P 668, 13.560-970, SÃo CARlos, SP, BrazIL

E-mail address: regilene@icmc.usp.br, camilaap@icmc.usp.br 